\title{
Inhibitory Effects of Pu-erh Tea Leaves on Mouse Type IV Allergy
}

\author{
Katsuko Yamazaki ${ }^{1}$, Kyoji Yoshino ${ }^{2}$, Chihiro Yagi $^{2}$, Toshio Miyase ${ }^{3}$, Mitsuaki Sano ${ }^{1}$ \\ ${ }^{1}$ Department of Food Science and Nutrition, Nagoya Women's University, Nagoya, Japan; ${ }^{2}$ Department of Chemistry and Biochem- \\ istry, Numazu National College of Technology, Shizuoka, Japan; ${ }^{3}$ School of Pharmaceutical Sciences, University of Shizuoka, Shi- \\ zuoka, Japan. \\ Email: sano@nagoya-wu.ac.jp
}

Received December $1^{\text {st }}$, 2011; revised January $17^{\text {th }}$, 2012; accepted January $25^{\text {th }}, 2012$

\begin{abstract}
Pu-erh tea is one of post fermented tea manufactured through oxidative maturing by microorganisms. We investigated the preventive effects of pu-erh tea extract on oxazolone-induced type IV allergy in male (ICR) mice. Oral administration of $50 \mathrm{mg} / \mathrm{kg}$ water extract of pu-erh tea leaves resulted in significant preventive effects against mouse type IV allergy. The hydrophilic Theabrownin-like fraction (TBW-ND), with a high molecular weight of approximately 12,000 or higher, was prepared from pu-erh tea leaves by a solvent-extraction method, followed by Diaion HP-20 column chromatography and dialysis. Oral and percutaneous administrations of TBW-ND at doses of $18.7 \mathrm{mg} / \mathrm{kg}$ and $0.037 \mathrm{mg} / \mathrm{ear}$ also resulted in significant preventive effects, which included preventing increases in levels of the proinflammatory cytokine interleukin-12. The anti-allergic ingredients in TBW-ND were predicted to be highly complex compounds containing of polyphenols, polysaccharides and/or proteins. The results suggest pu-erh tea leaves could be a beneficial food for protecting against delayed-type allergy.
\end{abstract}

Keywords: Pu-erh Tea; Type IV Allergy; Cytokines; Theabrownin; Polyphenol

\section{Introduction}

The increasing incidence of allergic diseases worldwide has become an important medical problem. Allergies can be divided into four types, types I-IV, on the basis of their mechanisms [1]. Type IV allergy, known as delayedtype hypersensitivity, is a cell-mediated immune reaction. Typical symptoms include contact dermatitis induced by proinflammatory cytokines released from type $1 \mathrm{~T}$ helper (Th1) cells.

Tea is one of the most popular beverages in the world, and various kinds of tea reflect differences in manufacturing processes. Green tea is classified as an unfermented tea, oolong tea as a partially fermented tea, black tea as a fully fermented tea and pu-erh tea as a post fermented tea. Pu-erh tea is a Chinese tea produced by the fermentation of green tea leaves with microorganisms such as Aspergillus species [2]. Pu-erh tea is usually preserved for a long period. It is believed that the longer preservation period is better for the quality of pu-erh tea. These features of the tea differ greatly from those of green tea, which is unfermented and is preferably taken as fresh as possible, Some reports, including our previous study, showed that pu-erh tea has an hypolipidemic and antiobesity effects in rat [3-5] and human [6]. These ef- fects are the same as those caused by green tea and green tea catechins [7-10], though the total content of monomeric catechins in pu-erh tea leaves is low (ca. 14\% of that in green tea leaves). Black tea leaves also possess lower levels of catechins, because catechins are oxidized enzymatically during the fermentation process to produce antioxidant polymers such as theaflavins, thearubigin and condensed theabrownins (TBs) [11-13]. Thearubigins, like catechins and theaflavins, have strong anti-inflammatory effects [14], while TBs are strongly oxidized and hydrophilic rust-brown products. The TB-like compounds in pu-erh tea leaves are water-soluble, but cannot be dissolved in ethyl acetate, n-butyl alcohol or other organic solvents, and show a significant hypocholesterolemic effect $[13,15,16]$. TB-like and thearubigin-like components and more polymerized brown pigments are also examples of polyphenols found in pu-erh tea leaves, though the total polyphenol content of pu-erh tea leaves is only about $54 \%$ that of green tea leaves [17].

We previously reported that an extract of green tea, catechins and their O-methylated derivatives in green and oolong tea leaves, theaflavins, dimers of catechins in black tea leaves and the C-2 epimers of catechins in tea infusions could prevent oxazolone-induced mouse ear 
edema [18-20]. These anti-allergic activities of tea catechins and theaflavins were thought to be due to their anti-inflammatory activities including antioxidant functions [21] and the inhibitory effects on the differentiation and proliferation of effector T cells [22].

There is currently no information on the anti-allergic effects of pu-erh tea; therefore, we investigated whether pu-erh tea leaves and their components protected against oxazolone-induced mouse type IV allergy. We also determined the effects of pu-erh tea leaves on cytokine levels in the ears of allergic mice.

\section{Materials and Methods}

\subsection{Reagents and Tea Samples}

Oxazolone(4-ethoxymethylene-2-phenyl-2-oxazolin-5-one) was purchased from Sigma-Aldrich Chemical Co. (Milwaukee, WI) and used as a sensitizer for type IV allergy. (-)-Epigallocatechin-3-O-gallate (EGCG) was purchased from Kurita Co. (Tokyo, Japan). Other chemicals used were of reagent grade.

The leaves of pu-erh tea and green tea (Yabukita cultivar) were purchased from a Chinese tea specialty store and supermarket in Japan, respectively. Water extracts of pu-erh tea leaves (PTE) and green tea leaves (GTE) were prepared as follows: tea samples (100 g) were extracted in $1.0 \mathrm{~L}$ of water at $70^{\circ} \mathrm{C}$ for $30 \mathrm{~min}$ and passed through filter paper. The filtrates were lyophilized, and PTE and GTE were obtained.

\subsection{Fractionation of Tea Extract}

A TB-like fraction of pu-erh tea was prepared according to the method of Gong et al. $[13,15]$ with minor modifycation. Briefly, pu-erh tea leaves (160 g) were extracted with methanol for $52 \mathrm{~h}$ and the methanol extract was discarded. The residue of tea leaves was extracted twice with distilled water for $3 \mathrm{~h}$ at $100^{\circ} \mathrm{C}$ and filtered. The resulting aqueous extract was washed with chloroform for $20 \mathrm{~h}$, ethyl acetate for $8 \mathrm{~h}$ and n-butanol three times, and the extract solutions were discarded. The residual aqueous solution was lyophilized and resolved in $400 \mathrm{~mL}$ of water. This solution was precipitated by $1.6 \mathrm{~L}$ ethanol, and the insoluble material lyophilized to obtain a TB-like fraction. This fraction was further fractionated by column chromatography with Diaion HP-20, a porous polymer gel (Mitsubishi Chemical Co. Tokyo, Japan). The size of the column was $3.2 \times 13 \mathrm{~cm}$. The aqueous solution of a TB-like fraction of $2.0 \mathrm{~g}$ was applied to the column, eluted first with $1.0 \mathrm{~L}$ water (producing TBW) and a second time using $50 \%(\mathrm{v} / \mathrm{v})$ methanol aqueous solution (producing TBM). The eluates were then lyophilized. TBM was slightly lipophilic, while TBW showed no lipophilicity. The aqueous solution of TBW was further dialyzed against an appropriate volume of water with a cellulose tube (exclusion molecular weight ca. 12,000, Wako Pure Chem. Ind., Osaka, Japan). The dialysate and non-dialysate were lyophilized as TBW-D and TBW-ND, respectively. The preparation procedure is shown in Figure 1.

\subsection{Mouse Type IV Allergic Model}

Oxazolone-induced edema of the mouse ear was used as a model of type IV allergy [23,24]. The sensitizer oxazolone was purchased from Wako Pure Chem. Ind. Male imprinting control region (ICR) mice, 4 weeks old, were purchased from Japan SLC, Inc. (Shizuoka, Japan). The hair of the mouse abdominal region was carefully shaved, and $0.1 \mathrm{~mL}$ of $0.5 \%$ oxazolone solution in ethanol was applied to the skin (sensitization). Five days after sensitization, $20 \mu \mathrm{L}$ of $0.5 \%$ oxazolone solution in acetone was applied to both sides of the right ear of each animal (challenge). Twenty-four hours after the challenge, the mice were sacrificed under ether anesthesia, and circular parts (5.0 mm in diameter) were removed from both ears using a punch. The weight of the right and left ears (WR and WL, respectively) were measured, and the ear swelling ratio was calculated using the following equation:

$$
\begin{aligned}
& \text { Ear swelling ratio }(\%) \\
& =\frac{\{(\text { WRsample }- \text { WLsample }) / \text { WLsample }\}}{\{(\text { WRcontrol }- \text { WLcontrol }) / \text { WLcontrol }\}} \times 100
\end{aligned}
$$

For oral administration, samples prepared from pu-erh and green tea leaves, EGCG and hydrocortisone were dissolved in $0.5 \%$ tragacanth gum solution, and $10-200$ $\mathrm{mg} / \mathrm{kg}$ was administered $1 \mathrm{~h}$ prior to the challenge. Hydrocortisone, a well-known steroid-type anti-inflammatory agent, was used as a positive control. Mice in the “Allergy” group were administered only $0.5 \%$ tragacanth gum solution. The body weight of mice in this experiment was $23.2 \pm 1.5 \mathrm{~g}$.

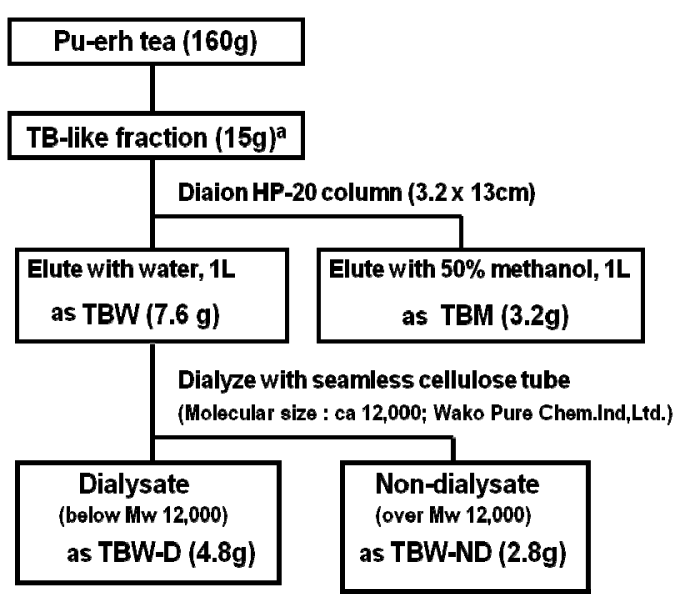

Figure 1. Preparation and fractionation of theabrownin (TB)-like fraction from pu-erh tea leaves. ${ }^{\mathrm{a}}$ TB-like fraction was prepared as described in method section. 
For percutaneous administration, samples prepared from pu-erh tea leaves and hydrocortisone were dissolved in oxazolone/acetone solution for the challenge and administered at doses of 0.037 - $0.2 \mathrm{mg} / \mathrm{ear}$. Mice in the "Allergy" group were administered only the solvents used for dissolution of the samples. The body weight of mice in this experiment was $22.4 \pm 2.6 \mathrm{~g}$.

The number of mice in each group was 5 . Throughout the experiment, the animals were handled in accordance with The Guide for the Animal Experiments in Numazu National College of Technology, which is based on "Fundamental Guidelines for Proper Conduct of Animal Experiment and Related Activities in Academic Research Institutions", under the jurisdiction of the Ministry of Education, Culture, Sports, Science and Technology of Japan.

\subsection{Determination of Interleukin-12 Levels in Mouse Ears}

The levels of interleukin(IL)-12 in mouse ears were determined by an enzyme-linked immunosorbent assay (ELISA) using a Mouse IL-12 Platinum ELISA kit (Bender MedSystems, Vienna, Austria).

\subsection{Determination of Polyphenol Contents and Related Compounds in Tea Samples}

The total contents of polyphenols including catechins and condensed tannins in PTE and GTE were determined by Folin-Denis' colorimetric method. Values were expressed as equivalent to ethyl gallate [25]. The levels of compounds consisting of catechin-based chemical structures in PTE, GTE, TBW, TBM, TBW-D and TBW-ND were assayed by a fluorometric method [26]. Fluorescence intensities of $0.1 \mathrm{mg} / \mathrm{mL}$ aqueous solutions were determined at an excitation wave length of $288 \mathrm{~nm}$ and an emission wave length of $319 \mathrm{~nm}$, which was a specific fluorometric characteristic of the catechin structure.

\subsection{Statistics}

Differences between the appropriate experimental groups were analyzed by one-way analysis of variance (ANOVA) followed by Tukey's multiple comparison test. P-values of $<0.05$ were considered statistically significant.

\section{Results and Discussion}

\subsection{Fractionation of Pu-erh Tea Extract}

PTE and GTE yields from tea leaves were 26.0\% and 17.3\%, respectively. As shown in Figure 1, the yields of the TB-like fraction, TBM, TBW, TBW-D and TBW-ND were $9.4 \%, 2.0 \%, 4.8 \%, 3.0 \%$ and $1.8 \%$, respectively. Previous yields of TB in black tea were reported as $4 \%$ $9 \%$, while those of TB-like fractions in pu-erh tea were $10 \%-14 \%$ [15]. Thus, the present TB-like fraction yield almost matched the previously reported range.

\subsection{Effects of PTE Oral Administration on Mouse Type IV Allergy and IL12 Ear Levels}

The preventive effects of PTE oral administration against type IV allergy in ICR mice were compared with those of GTE and EGCG (Figure 2(a)). The administration of hydrocortisone and EGCG strongly suppressed the allergic reaction at doses of 100 and $50 \mathrm{mg} / \mathrm{kg}$, respectively. The administration of $50 \mathrm{mg} / \mathrm{kg}$ PTE and GTE also significantly prevented mouse type IV allergy, though no efficacy was observed following the administration of 10 $\mathrm{mg} / \mathrm{kg}$ PTE. The effects of PTE oral administration on IL-12 levels in the ears of mice with oxazolone-induced type IV allergy were compared with those of GTE and EGCG (Figure 2(b)). Ear IL-12 levels rose significantly

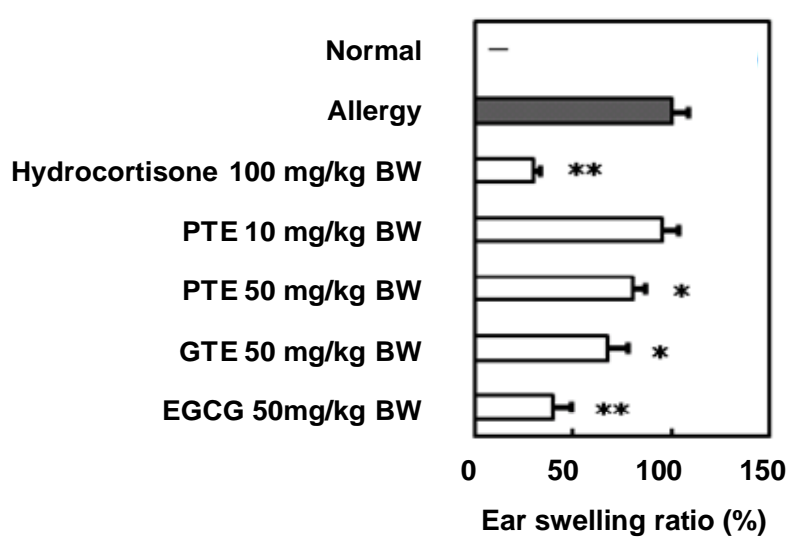

(a)

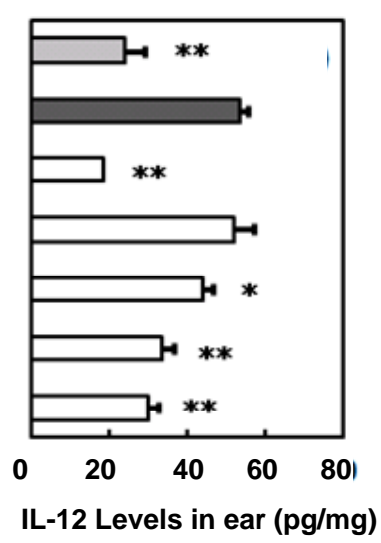

(b)

Figure 2. Preventive effects of oral administered pu-erh tea water extract on mouse type IV allergy. (a) Ear swelling ratio (\%); (b) Interleukin-12 levels in ear (pg/mg). Mean \pm SE. $n=5$. Significant differences from "Allergy" group; $* P<0.05, * * P<0.01$. PTE, water extract of pu-erh tea; GTE, water extract of green tea; EGCG, (-)-epigallocatechin gallate. 
in mice with severe allergic symptoms, such as inflamemation, compared with normal mice. However, the oral administration of $100 \mathrm{mg} / \mathrm{kg}$ hydrocortisone or $50 \mathrm{mg} / \mathrm{kg}$ PTE, GTE and EGCG significantly prevented this increase in mouse ears.

These preventive activities on the increase of IL-12 levels in mouse ears accorded with those on mouse type IV allergic symptoms. IL-12 is secreted by macrophagelike antigen presenting cells and induces differentiation of naive T cells into Th1 cells. Our results therefore indicate that infusions of pu-erh tea leaves could be used in the same way as those of green tea leaves to prevent type IV allergies. The suppressive effect of PTE on the induction of proinflammatory cytokines is thought to contribute to the prevention of type IV allergies in mice.

We previously reported that pu-erh tea leaf components were of higher molecular weight than components of other kinds of tea leaves, had a low content of monomeric catechins, retained their polyphenolic properties and were comparatively highly hydrophilic [17]. The PTE polyphenol content was $27.4 \% \pm 0.7 \%(\mathrm{w} / \mathrm{w})$ as ethyl gallate equivalents, while that of GTE was $49.5 \% \pm$ $1.0 \%(\mathrm{w} / \mathrm{w})$. Pu-erh tea also includes brown pigments like thearubigin and TB [15-17]. Black tea thearubigin consists of a mixture of polyphenols, amino acids and polysaccharides and has a molecular weight of 700 40,000 [27]. Black tea TB is formed by further polymerization of theaflavins, thearubigin and other polyphenols [11]. The TB-like fraction of pu-erh tea leaves consists of $3.54 \%$ conjugated polysaccharides and $12.1 \%$ conjugated proteins [15]. In the present study, we hypothesized that polyphenol complexes such as the TB-like fraction would be the main active components responsible for the anti-allergic effects of pu-erh tea leaves. We therefore analyzed the effects of fractions prepared from TB-like fractions of pu-erh tea leaves.

\subsection{The Effects of Orally Administered Fractions Prepared from the TB-like Fraction of Pu-erh Tea Leaves}

The effects of the oral administration of TBM, TBW, TBW-D and TBW-ND against type IV allergy in ICR mice are shown in Figure 3(a). The non-lipophilic TBW and slightly lipophilic TBM fractions, separated by a Diaion HP-20 column, were administered at $50 \mathrm{mg} / \mathrm{kg}$. TBM showed no preventive effects while TBW significantly prevented the development of allergic symptoms. Indeed, TBW showed dose-dependent preventive effects against mouse type IV allergy at doses of 50, 100 and $200 \mathrm{mg} / \mathrm{kg}$. As the yield of TBW from pu-erh tea leaves was approximately twice as high as that of TBM, we hypothesized that it is the main active fraction responsible for the anti-allergic effects of the TB-like fraction of pu-erh tea leaves.

Since an efficient anti-allergic effect was observed following the administration of TBW, it was dialyzed further to obtain a lower molecular weight fraction, TBW-D, and a higher molecular weight fraction, TBWND. The exclusion molecular weight was approximately 12,000 . We examined the effects of an oral administration of TBW-D and TBW-ND at doses equivalent to those yielded from $50 \mathrm{mg}$ TBW/kg body weight, 31.3 and $18.7 \mathrm{mg} / \mathrm{kg}$ body weight, respectively. Only TBWND administration produced a significant anti-allergic effect. For this reason, it appears that one of the main active components responsible for the anti-allergic effects of pu-erh tea leaves is a hydrophilic ingredient with a molecular weight of about 12,000 or higher.

The effects of an oral administration of TBM, TBW, TBW-D and TBW-ND on IL-12 levels in the ears of mice with oxazolone-induced type IV allergy are shown in Figure 3(b). Similar to the effects on mouse allergic symptoms, the administration of $50 \mathrm{mg} / \mathrm{kg} \mathrm{TBM}$ and $31.3 \mathrm{mg} / \mathrm{kg}$ TBW-D had no preventive effects on the increase in IL-12 levels. However, TBW showed dosedependent preventive effects at doses of 50, 100 and 200 $\mathrm{mg} / \mathrm{kg}$. The administration of $18.7 \mathrm{mg} / \mathrm{kg}$ TBW-ND also significantly suppressed the IL-12 increases. The antiallergic effects of orally administered TBW and TBWND could therefore partly be caused by their suppressive effects on IL-12 increases in mouse ears.

\subsection{The Effects of Percutaneously Administered Fractions Prepared from the TB-Like Fraction of Pu-erh Tea Leaves}

As some fractions prepared from the TB-like fraction of pu-erh tea leaves showed preventive effects on mouse type IV allergy, we next determined whether the direct application of these fractions on mouse ears could prevent contact dermatitis. The effects of the percutaneous administration of TBM, TBW, TBW-D and TBW-ND against type IV allergy in ICR mice are shown in Figure 4(a). TBM administration resulted in no preventive effects against mouse type IV allergy at doses of 0.1 mg/ear, though the administration of TBW significantly prevented contact dermatitis at the same dose. Moreover, a dose-dependent preventive effect was observed following TBW administration at doses of 0.1 and 0.2 mg/ear. No preventive effect was observed following $0.05 \mathrm{mg} / \mathrm{ear}$ TBW administration. When TBW-D and TBW-ND were percutaneously administered at doses equal to those yielded from $1.0 \mathrm{mg}$ TBW/ear, 0.063 and $0.037 \mathrm{mg} / \mathrm{ear}$, respectively, only TBW-ND showed significant anti-allergic effects. This is consistent with our earlier finding regarding oral administration that a hydrophilic ingredient of molecular weight over ca. 12,000 


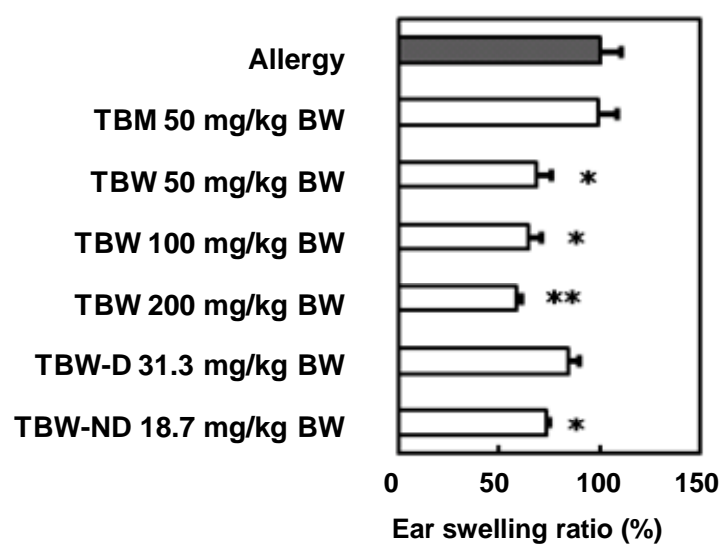

(a)

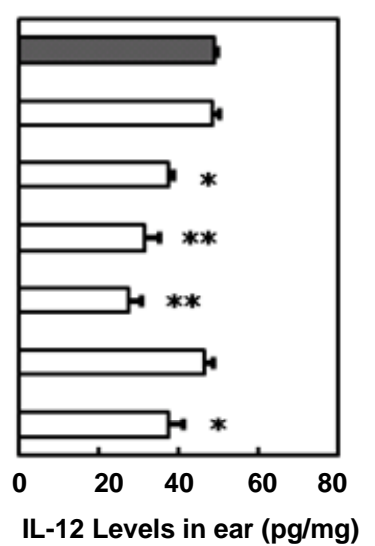

(b)

Figure 3. Preventive effects of oral administered fractions prepared from TB-like fraction of pu-erh tea on mouse type IV allergy. (a) Ear swelling ratio (\%); (b) Interleukin-12 level in ear (pg/mg). Mean \pm SE. $n=5$. Significant differences from “Allergy" group; ${ }^{*} \boldsymbol{P}<0.05,{ }^{*} \boldsymbol{P}<0.01$. TBM, methanol eluate of TB-like fraction through Diaion HP-20 column; TBW, water eluate of TB-like fraction through Diaion HP-20 column; TBW-D, dialysate of TBW; TBW- ND, non-dialysate of TBW.

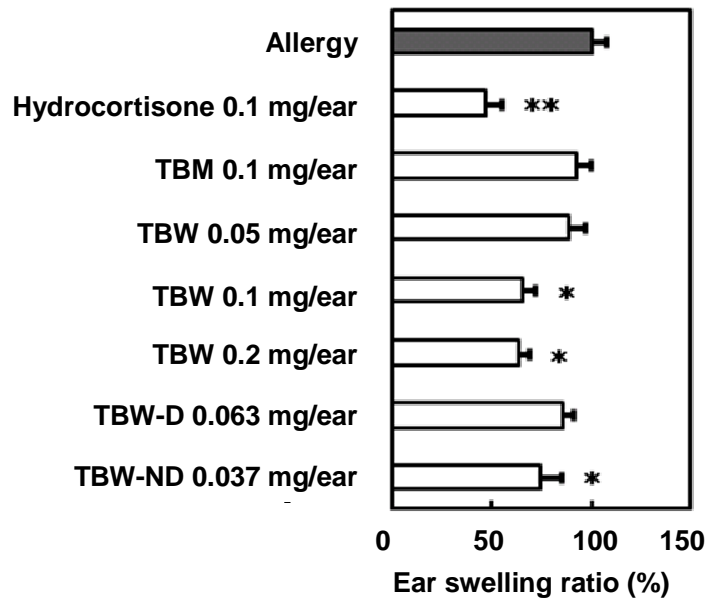

(a)

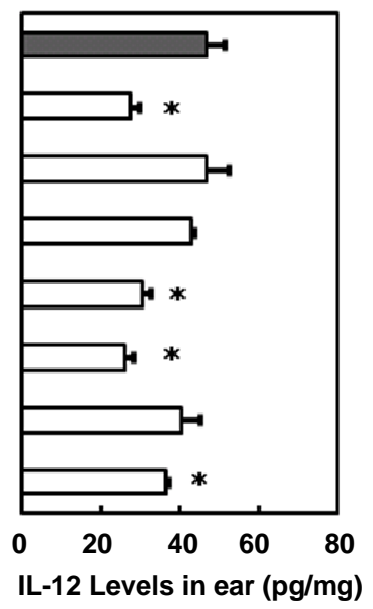

(b)

Figure 4. Preventive effects of percutaneously administered fractions prepared from TB-like fraction of pu-erh tea on mouse type IV allergy. (a) Ear swelling ratio (\%); (b) Interleukin-12 level in ear (pg/mg). Mean \pm SE. $n=5$. Significant differences from "Allergy" group; $* P<0.05, * * P<0.01$. TBM, methanol eluate of theabrownin-like fraction through Diaion HP-20 column; TBW, water eluate of theabrownin-like fraction through Diaion HP-20 column; TBW-D, dialysate of TBW; TBW-ND, non-dialysate of TBW.

appears to be one of the main active components.

The effects of the percutaneous administration of TBM, TBW, TBW-D and TBW-ND on IL-12 levels in the ears of mice with oxazolone-induced type IV allergy are shown in Figure 4(b). Fluctuations in IL-12 levels within mouse ears were in accordance with ear swelling ratios between groups. The significant lowering effects of percutaneously administered TBW at doses of 0.1 and 0.2 mg/ear and TBW at $0.037 \mathrm{mg} / \mathrm{ear}$ are thought to contribute to their anti-allergic effects. In both cases of oral and percutaneous administration, the active fractions of pu-erh tea leaves and their partial mechanisms of anti-allergic effects appeared to be similar.

Fluorescence intensities were determined by the levels of compounds containing catechin-based chemical structures from aqueous solutions of GTE, PTE, TBM, TBW, TBW-D and TBW-ND (Figure 5). GTE contained more catechin-based compounds than PTE, and TBW contained more than TBM, which mirrored the differences in intensity of their anti-allergic effects. However, the level of catechin-based compounds in PTE was higher than that of TBW, even though the anti-allergic effect of PTE was weaker than TBW. The same was true for TBW-D and TBW-ND which showed higher catechin-based compound levels but weaker anti-allergic effects. These results suggest that particular polyphenols and other unknown components are involved in the preventive effects of PTE, TBW and TBW-ND on mouse type IV allergy. 


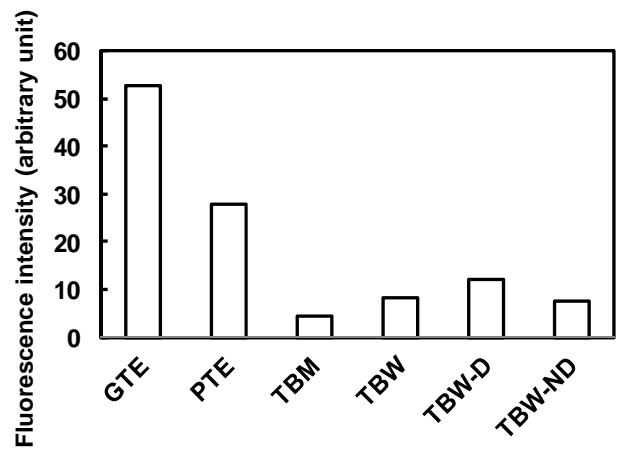

Figure 5. Fluorescence intensities of aqueous solutions (0.1 $\mathrm{mg} / \mathrm{mL}$ ) of fractions prepared from pu-erh and green tea leaves as the levels of compounds consisting of cate-chinbased chemical structures. Data are represented as means of duplicate measurements using each fractionated sample.

We confirmed that the content of EGCG, a major monomeric catechin in tea leaves, was very low in TBW, at $0.001 \%(\mathrm{w} / \mathrm{w})$. In addition to polyphenols, saponins and polysaccharide complexes such as glycoproteins are known anti-allergic components of green tea leaves $[28,29]$. The molecular weight of saponins and glycoproteins in tea leaves is about. 1300 and 110,000 , respectively. The anti-allergic ingredients in TBW-ND from pu-erh tea leaves could therefore be predicted to be highly complex compounds consisting of polyphenols, polysaccharides and/or proteins. Further investigations about several partial chemical structures of the ingredients in TBW-ND from pu-erh tea leaves would be available to clear their whole chemical structures like as the study for the determination of chemical structures of condensed tannin in persimmon (Diospyros kaki) [30].

\section{Conclusion}

Oral and percutaneous administrations of pu-erh tea infusions and the highly hydrophilic high molecular weight fraction TBW-ND showed potent preventive effects on oxazolone-induced type IV allergy in male ICR mice, at least partially through inhibiting the production and/or release of IL-12 from macrophages. TBW-ND is a specific component of pu-erh tea leaves and its active components appear to be complexes consisting of catechins, polysaccharides and/or proteins. Our findings suggest that pu-erh tea leaves could be a beneficial food for protecting against type IV allergy contact hypersensitivity.

\section{REFERENCES}

[1] T. V. Rajan, "The Gell-Coombs Classification of Hypersensitivity Reactions: A Re-Interpretation," Trends in Immunology, Vol. 24, No. 7, 2003, pp. 376-379. doi:10.1016/S1471-4906(03)00142-X

[2] J. H. Clarke, J. M. Nayor, J. N. Banks, K. A. Scudamore, Y. Ito, T. Mukai, H. Horie and T. Goto, “The Mycofloras and Potential for Mycotoxin Production of Various Samples of Green, Fermented and Late Fermented Tea,” Tea Research Journal, Vol. 79, No. 1, 1994, pp. 31-36. doi:10.5979/cha.1994.31

[3] M. Sano, Y. Takenaka, R. Kojima, S. Saito, I. Tomita and M. Katou, "Effects of Pu-erh Tea on Lipid Metabolism in Rats,” Chemical \& Pharmaceutical Bulletin, Vol. 34, No. 1, 1986, pp. 221-228. doi:10.1248/cpb.34.221

[4] T. T. Yang and M. W. Koo, "Hypocholesterolemic Effects of Chinese Tea,” Pharmacological Research, Vol. 35, No. 6, 1997, pp. 506-512. doi:10.1006/phrs.1997.0176

[5] Z. H. Cao, D. H. Gu, Q. Y. Lin, Z. Q. Xu, Q. C. Huang, H. Rao, E. W. Liu, J. J. Jia and C. R. Ge, "Effect of Pu-erh Tea on Body Fat and Lipid Profiles in Rats with DietInduced Obesity,” Phytotherapy Research, Vol. 25, No. 2, 2011, pp. 234-238.

[6] K. Kubota, S. Sumi, H. Tojo, Y. Sumi-Inoue, H. I. Chin, Y. Oi, H. Fujita and H. Urata, "Improvements of Mean Body Mass Index and Body Weight in Preobese and Overweight Japanese Adults with Black Chinese Tea (Pu-erh) Water Extract,” Nutrition Research, Vol. 31, No. 6, 2011, pp. 421-428. doi:10.1016/j.nutres.2011.05.004

[7] J. K. Lin and S. Y. Lin-Shiau, "Mechanisms of Hypolipidemic and Anti-Obesity Effects of Tea and Tea Polyphenols," Molecular Nutrition \& Food Research, Vol. 50, No. 2, 2006, pp. 211-217. doi:10.1002/mnfr.200500138

[8] M. Bose, J. D. Lambert, J. Ju, K. R. Reuhl, S. A. Shapses and C. S. Yang, “The Major Green Tea Polyphenol, (-)-Epigallocatechin-3-gallate, Inhibits Obesity, Metabolic Syndrome, and Fatty Liver Disease in High-Fat-Fed Mice,” Journal of Nutrition, Vol. 138, No. 9, 2008, pp. 1677-1683.

[9] T. Nagao, T. Hase and I. Tokimitsu, "A Green Tea Extract High in Catechins Reduces Body Fat and Cardiovascular Risks in Humans,” Obesity, Vol. 15, No. 6, 2007, pp. 1473-1483. doi:10.1038/oby.2007.176

[10] P. Auvichayapat, M. Prapochanung, O. Tunkamnerdthai, B. O. Sripanidkulchai, N. Auvichayapat, B. Thinkhamrop, S. Kunhasura, S. Wongpratoom, S. Sinawat and P. Hongprapas, "Effectiveness of Green Tea on Weight Reduction in Obese Thais: A Randomized, Controlled Trial," Physiology \& Behavior, Vol. 93, No. 3, 2008, pp. 486-491. doi:10.1016/j.physbeh.2007.10.009

[11] L. H. Yao, Y. M. Jiang, N. Caffin, B. D’Arcy, N. Datta, $\mathrm{X}$. Liu, R. Singanusong and Y. Xu, "Phenolic Compounds in Tea from Australian Supermarkets,” Food Chemistry, Vol. 96, No. 4, 2006, pp. 614-620. doi:10.1016/j.foodchem.2005.03.009

[12] Y. Ziyin, T. U. Youying, J. Guoliang, C. Xiaoming, H. E. Puming and $\mathrm{X}$. Huilong, "Suppression of Free-Radicals and Protection against $\mathrm{H}_{2} \mathrm{O}_{2}$-Induced Oxidative Damage in HPF-1 Cell by Oxidized Phenolic Compounds Present in Black Tea,” Food Chemistry, Vol. 105, No. 4, 2007, pp. 1349-1356. doi:10.1016/j.foodchem.2007.05.006

[13] J. Gong, W. Chen, H. Zhou, Z. Dong and Y. Zhang, "Evaluation on the Function and Toxicity of Extraction of Characteristic Components in Yunnan Pu-erh Tea," Journal of tea Sciences, Vol. 27, No. 3, 2007, pp. 201- 
210.

[14] S. Maity, A. Ukil, S. Karmakar, N. Datta, T. Chaudhuri, J. R. Vedasiromoni, D. K. Ganguly and P. K. Das, "Thearubigin, the Major Polyphenol of Black Tea, Ameliorates Mucosal Injury in Trinitrobenzene Sulfonic Acid-Induced Colitis,” European Journal of Pharmacology, Vol. 470, No. 1-2, 2003, pp. 103-112. doi:10.1016/S0014-2999(03)01760-6

[15] J. Gong, C. Peng, T. Chen, B. Gao and H. Zhou, "Effects of Theabrownin from Pu-erh Tea on the Metabolism of Serum Lipids in Rats: Mechanism of Action,” Journal of Food Science, Vol. 75, No. 6, 2010, pp. H182-H189. doi:10.1111/j.1750-3841.2010.01675.x

[16] Q. Wang, C. Peng and J. Gong, "Effects of Enzymatic Action on the Formation of Theabrownin during Solid State Fermentation of Pu-erh Tea," Journal of the Science of Food and Agriculture, Vol. 91, No. 13, 2011, pp. 2412-2418. doi:10.1002/jsfa.4480

[17] K. Yoshino, M. Aoki, H. Ikeya, C. Urashima and I. Tomita, "Pattern Analysis of Various Tea Brown Pigments through Sephadex LH-20 Column Chromatography,” Japanease Journal of Food Chemistry, Vol. 2, No. 1, 1995, pp. 9-13.

[18] M. Suzuki, K. Yoshino, M. Maeda-Yamamoto, T. Miyase and M. Sano, "Inhibitory Effects of Tea Catechins and O-methylated Derivatives of (-)-Epigallocatechin-3-Ogallate on Mouse Type IV Allergy,” Journal of Agricultural and Food Chemistry, Vol. 48, No. 11, 2000, pp. 5649-5653. doi:10.1021/jf000313d

[19] K. Yoshino, K. Ogawa, T. Miyase and M. Sano, "Inhibitory Effects of the C-2 Epimeric Isomers of Tea Catechins on Mouse Type IV Allergy,” Journal of Agricultural and Food Chemistry, Vol. 52, No. 11, 2004, pp. 46604663. doi:10.1021/jf035476r

[20] K. Yoshino, K. Yamazaki and M. Sano, "Preventive Effects of Black Tea Theaflavins against Mouse Type IV Allergy," Journal of the Science of Food and Agriculture, Vol. 90, No. 12, 2010, pp. 1983-1987.

[21] F. Nanjo, M. Mori, K. Goto and Y. Hara, "Radical Scavenging Activity of Tea Catechins and Their Related Compounds," Bioscience, Biotechnology, and Biochemistry, Vol. 63, No. 9, 1999, pp. 1621-1623. doi:10.1271/bbb.63.1621
[22] M. Das, P. Sur, A. Gomes, J. R. Vedasiromoni and D. K. Ganguly, "Inhibition of Tumour Growth and Inflammation by Consumption of Tea," Phytotherapy Research, Vol. 16, Suppl. 1, 2002, pp. 40-44. doi:10.1002/ptr.797

[23] Y. Nakano, "Antigenic Competition in the Induction of Contact Sensitivity in Mice,” Immunology, Vol. 33, No. 2, 1977, pp. 167-178.

[24] H. Nakamura, S. Motoyoshi, K. Ishii, Y. Seto and M. Shimizu, "Anti-Inflammatory Activity of a Topical Glucocorticoid, Fludroxycortide Tape in Experimental Animals,” Folia Pharmacologica Japonica, Vol. 76, No. 7, 1980, pp. 595-607. doi:10.1254/fpj.76.595

[25] O. Folin and W. Denis, “A Colorimetric Method for the Determination of Phenols (and Phenol Derivatives) in Urine,” The Journal of Biological Chemistry, Vol. 22, No. 2, 1915, pp. 305-308.

[26] I. Ramirez-Sanchez, L. Maya, G. Ceballos and F. Villarreal, "Fluorescent Detection of (-)-Epicatechin in $\mathrm{Mi}$ crosamples from Cacao Seeds and Cocoa Products, Comparison with Folin-Ciocalteu Method," Journal of Food Composition and Analysis, Vol. 23, No. 8, 2010, pp. 790-793. doi:10.1016/j.jfca.2010.03.014

[27] D. J. Millin, D. Swaine and P. L. Dix, "Separation and Classification of the Brown Pigments of Aqueous Infusions of Black Tea," Journal of the Science of Food and Agriculture, Vol. 20, No. 5, 1969, pp. 296-302. doi:10.1002/jsfa.2740200511

[28] M. Akagi, N. Fukuishi, T. Kan, Y. M. Sagesaka and R. Akagi, "Anti-Allergic Effect of Tea-Leaf Saponin (TLS) from Tea Leaves (Camellia sinensis var. sinensis)," Biological \& Pharmaceutical Bulletin, Vol. 20, No. 5, 1997, pp. 565-567. doi:10.1248/bpb.20.565

[29] W. Dongfeng, W. Chenghong, L. Jun and Z. Guiwen, "Components and Activity of Polysaccharides from Coarse Tea,” Journal of Agricultural and Food Chemistry, Vol. 49, No. 1, 2001, pp. 507-510. doi:10.1021/jf000029m

[30] T. Matsuo, K. Tamaru and S. Ito, “Chemical Degradation of Condensed Tannin with Phloroglucinol in Acidic Solvents," Agriculture and Biological Chemistry, Vol. 48, No. 5, 1984, pp. 1199-1204. doi:10.1271/bbb1961.48.1199 